\title{
Mutations in conserved regions of eel luteinizing hormone receptor result in constitutive activation or inactivation
}

Munkhzaya Byambaragchaa ${ }^{1}$, Dong-An Kim ${ }^{1}$, Dae-Jung Kim ${ }^{2}$, Sun-Mee Hong ${ }^{3}$, Myung-Hwa $\mathrm{Kang}^{4}$ and Kwan-Sik Min ${ }^{1, *}$

${ }^{1}$ Animal Biotechnology, Graduate School of Future Convergence Technology, Department of Animal Life Science, Institute of Genetic Engineering, Hankyong National University, Ansung 17579, Korea

E-Mail: monkhzaya_b@yahoo.com

E-Mail:wrm032@daum.net

${ }^{2}$ Jeju Fisheries Research Institute, National Institute of Fisheries Science (NIFS), Jeju 63610, Korea

E-Mail: djkim4128@korea.kr

${ }^{3}$ Dept. of Research and Development, Institute of Gyeongbuk Marine Bioindustry, Ulgin 36315, Korea

E-Mail: hongsunmee@gimb.or.kr

${ }^{4}$ Department of Food Science and Nutrition, Hoseo University, Asan 31499, Korea,

E-Mail: mhkang@hoseo.ac.kr

* Author to whom correspondence should be addressed; E-mail address: ksmin@hknu.ac.kr,

Tel.: +82-31-670-5421; Fax: +82-670-5417 
Abstract: Luteinizing hormone receptor (LHR) is a member of the seven-transmembrane (TM) receptor family. Several mutations in LHR have been identified in many mammalian species, leading to either constitutive activation or inactivation of the receptor. Mutations in highly conserved regions of the TM domain have been reported. In this study, we analyzed signal transduction by three constitutively active mutants (designated M410T, L469R, and D590Y) and two inactivating mutants (D383N and Y546F) of eelLHR known as naturally occurring in human LHR . To directly assess the functional effects of these mutations, site-directed mutant receptors were transiently expressed in $\mathrm{CHO}-\mathrm{K} 1$ cells and cAMP accumulation stimulated by recombinant eelLH (rec-eelLH) was measured by homogeneous time-resolved fluorescence (HTRF) assays. The cAMP response in cells expressing eelLHR wild-type (eLHR-WT) increased in a dosedependent manner with rec-eelLH ligand stimulation. Cells expressing the activating eelLHR mutants, M410T, L469R, and D590Y, exhibited a 4.0-, 19.1-, and 7.8-fold increase in basal cAMP response, respectively. However, their maximal responses were approximately 73, 53, and 92\%, respectively, of the maximal response of LHR-WT. The L469R mutant exhibited a particularly marked increase in cAMP production in the absence of agonist. The maximal responses of the inactivating mutants, D383N and Y546F, were 32 and 24\% of LHR-WT, respectively. However, the inactivating mutations did not completely impair signal transduction. Thus, we report here the first characterization of activating and inactivating mutations in eelLHR and we show that these mutations have similar effects as those reported for mammalian LHRs. Moreover, eelLHR with activating mutations showed constitutive cAMP responses. These results provide important data on the signal transduction of constitutively active and inactive LHR mutants. Further studies should aim to identify the mechanism responsible for the significant increase in basal cAMP response in the constitutively activated eelLHR mutants.

Key words: eel luteinizing hormone receptor, constitutively activating mutation, inactivating mutation, cAMP responses. 


\section{Introduction}

The seven-transmembrane G protein-coupled receptors (GPCR) represent one of the largest gene families in eukaryotic organisms [1]. Receptors for luteinizing hormone (LH) and folliclestimulating hormone (FSH) form a subgroup of glycoprotein hormone receptors within the GPCR family [2,3].

There is a great abundance of naturally occurring mutations in the LH receptor (LHR) gene that are related with reproductive failures in mammals [4]. The elucidation of the cDNA and genomic sequences of the human LH receptor (hLHR) have made it possible to identify hLHR mutations that can be directly connected to particular reproductive disorders [5-8]. Several dominant gain-of-function mutations in the hLHR gene have been identified in males with sporadic or familial male-limited gonadotropin-independent pseudo-precocious puberty, also known as "testotoxicosis" $[9,10]$. In the presence of a heterozygous activating mutation, boys with this condition display elevated levels of testosterone, but prepubertal levels of gonadotropin-releasing hormone $(\mathrm{GnRH})$ and LH [6,11], suggesting that the LHR-signaling pathway in their Leydig cells is activated, even in the absence of hormonal stimulation. These activating hLHR mutations also result in markedly increased cAMP production in the absence of agonist and this is the most common cause of male-limited precocious puberty $[12,13]$.

Cells expressing the hLHR mutant, D578Y, exhibit markedly increased cAMP production in the absence of agonist [6]. Such constitutive activity of the hLHR gene causes LH-releasing hormone-independent precocious puberty in boys and the autosomal dominant disorder, familial male-limited precocious puberty (FMPP) [8]. The Asp578 residue of hLHR serves as an appropriately positioned hydrogen bond acceptor to help stabilize the inactive state of the receptor [12]. An M398T hLHR mutant has been shown to cause constitutively high basal cAMP levels, consistent with Leydig cell activation and precocious puberty in the patient [7]. In a study of genomic DNA from 32 unrelated FMPP patients, 3 activating mutation sites (Asp578Gly, Met571Ile, and Thr577Ile) and 4 other mutation sites (Ile542Leu, Asp564Gly, Asp578Tyr, and Cys581Arg) were identified in hLHR [14,15]. The region spanning nucleotides 1624-1741 of exon 11 is a hotspot for heterogeneous point mutations that constitutively activate hLHR [13]. Germline hLHR mutations that display constitutive activation of the Gs pathway have also been found in cases of Leydig cell hyperplasia [11]. 
Another LH receptor mutation, M398T, in the second transmembrane helix, has been reported in a precocious puberty patient and in the patient's mother and brother [16,17]. This mutation is of special interest, since one member of the family has the mutation, with no evidence of precocious puberty [18]. Latronico et al. [9] first identified the hLHR activating mutation, L457R, and showed that cells expressing this mutant exhibited significantly higher basal levels of cAMP (7- to 14-fold) than cells expressing the LHR wild-type (LHR-WT). A potent activating somatic mutation $(\mathrm{D} 578 \mathrm{H})$ has also been identified in boys with testicular adenomas $[19,20]$. The D556H mutation in rat LHR (rD556H; equivalent to the $\mathrm{hD} 578 \mathrm{H}$ mutation) also results in an increase in the basal levels of cAMP [4]. A large number of constitutively activating mutations in LHR have been found in boys with sporadic or more common forms of FMPP [21,22].

The inactivating LHR mutations, D383N and $\mathrm{R} 442 \mathrm{H}$, do not affect human chorionic gonadotropin (hCG) binding, but they do impair signal transduction [23]. However, the cAMP responsiveness of the $\mathrm{D} 383 \mathrm{~N}$ and $\mathrm{R} 442 \mathrm{H}$ mutant receptor was characterized by an 18- and 7-fold increase in $\mathrm{EC}_{50}$, respectively, compared to the $\mathrm{EC}_{50}$ of an equivalent density of rLHR-WT [23]. We have also previously reported that L435R and D556Y mutations induce constitutive receptor activation and result in a 25- and 47-fold increase in basal cAMP responsiveness, respectively [24]. These mutations also enhance the internalization of free and agonist-occupied receptors by 2- and $\sim 17$-fold, respectively. The $\mathrm{D} 383 \mathrm{~N}$ and $\mathrm{Y} 524 \mathrm{~F}$ mutations are signal impairing mutations and show low-to-normal levels of cAMP under basal conditions. Thus, there are two known signal-impairing mutants that decrease the rate of hCG internalization [24]. All inactivating mutations result in impaired hCG-stimulated cAMP production in cells expressing the mutant receptors, suggesting a clear correlation between the severity of the clinical phenotype and overall receptor signal capacity, which reflects both cell-surface expression levels and coupling efficiency [15].

Although the hormone-binding and activation properties of these mutants are generally well characterized in hLHR, little is known about signal transduction in fish LHR. In the present study, we examined the effects of several constitutively activating (M410T, L469R, and D576Y) and inactivating mutations (D383N and Y546F) in highly conserved residues of eelLHR. We report here that the basal cAMP response of constitutively active eelLHR mutants and the maximal cAMP levels of the inactive mutants differed from those of eelLHR-WT. 


\section{Results}

\subsection{Construction of eelLHR mutants and Constitutively active mutant receptors}

eelLHR consists of 2,115 nucleotides encoding 705 amino acids. In order to generate substitution mutations at target amino acids, an overlap extension PCR strategy was used with primers designed to change target nucleotides (Table 1). We generated three constitutively activating mutations in transmembrane helices II, III, and VI to investigate how they affect the hormone-receptor interaction and receptor activation system. These mutant receptors were designated M410T (equivalent to M398T in hLHR), L469R, and D590Y. We also constructed two constitutively inactive mutants of eelLHR, designated as D383N and Y546F. Cells transfected with eelLHR-WT exhibited an increased production of cAMP in response to a high concentration of rec-eelLH, when $10^{4}$ cells were analyzed. The $\mathrm{EC}_{50}$ of the rec-eelLH-stimulated cAMP response was approximately $24 \mathrm{ng} / \mathrm{mL}$. The basal and Rmax cAMP responses were 1.2 and $96 \mathrm{nM} / 10^{4}$ cells, respectively.

To assess the functional effects of the three receptor mutations directly, eelLHR-WT and constitutively active mutant receptors were transiently expressed in CHO-K1 cells. Cells transfected with eelLHR-WT DNA had very low basal cAMP production. In contrast, cells expressing the constitutive active mutants (M410T, L469R, D590Y) had highly increased basal cAMP accumulation that was further respond by rec-eelLH in a dose-dependent manner. It was $4.8 \pm 0.3,22.9 \pm 1.5$, and $9.3 \pm 0.8 \mathrm{ng} / 10^{4}$ cells, respectively. Cells expressing eelLHR-L469R and -D590Y exhibited a 19.1- and 7.8-fold increase in basal cAMP accumulation respectively, compared to cells expressing eelLHR-WT. The EC50 values of these mutants for rec-eelLHstimulated cAMP production were 15 and $827 \%$ of LHR-WT values, respectively and their maximum cAMP production was lower than that of the eelLHR-WT. However, the M410T mutant showed only a 4-fold increase in basal cAMP production. The $\mathrm{EC}_{50}$ level and Rmax values for this mutant were only $20 \%$ and $73 \%$, respectively, of the values of eelLHR-WT. The maximum level of cAMP production of all the constitutively active mutants was lower than that of eelLHR-WT. Compared with eelLHR-WT, all eelLHRs with activating mutations produced higher basal levels of cAMP in CHO-K1 cells than eelLHR-WT, which is consistent with constitutive activation (Figure 1 and Table 2). The mutant with the highest level of basal cAMP production (L457R) did not react to rec-eelLH with a further increase in cAMP responsiveness. 
The maximum levels of cAMP production were the lowest in eelLHRs with activating mutations. Thus, high basal cAMP production was not consistent with cAMP responsiveness to agonist stimulation.

\subsection{Characterization of inactivating mutations}

The activity of eelLHRs with the inactivating mutations, D383N and Y546F, was measured by quantifying cAMP stimulation in cells incubated with increasing concentrations of rec-eelLH (Figure 2 and Table 2). As predicted, signaling was impaired in both mutant receptors. The $\mathrm{EC}_{50}$ for the rec-eelLH-induced increase in cAMP levels was 2.1-fold higher in the D383N mutant than in eel-LHR-WT. However, the maximal response of this manner was only $32 \%$ of the response of eelLHR-WT. In the Y546F mutant, the $\mathrm{EC}_{50}$ value was $67.2 \%$ of the value for eelLHR-WT, but the maximal response was only $24 \%$ lower than that of eel-LHR-WT. Basal cAMP levels in cells expressing D383N and Y546F mutants were 1.2 and $0.7 \mathrm{nM}$ respectively, which were slightly lower than the basal cAMP levels in cells expressing eelLHR-WT. As shown in Table 2, the rec-eelLH responsiveness of receptor was severely affected by the inactivating mutations. The maximal response of these cells was $68 \sim 76 \%$ lower than the maximal response of cells expressing eelLHR-WT.

\section{Discussion}

The present study describes mutations that induce constitutive activation or impaired signal transduction in eelLHR consistent with previously reported mammalian LHR mutations that cause FMPP and elevated cAMP levels in the absence of agonist. Thus, we prepared mutant eelLHRs containing single point mutations in five distinct amino acid residues that are highly conserved among GPCRs. These mutations have been shown to stimulate basal cAMP responsiveness or attenuate agonist-induced activation of other GPCRs.

Many studies have suggested that the similar active conformation of mammalian LHRs is involved in the stimulus of $\mathrm{G}$ proteins $[5,12,24]$. In humans, mutations have previously been described that constitutively activate hLHR and cause mutation of FMPP $[9,13]$. The differences observed in the phenotypic appearance of FMPP may be accounted by distinctions in basal hLHR activity [13]. In a previously reported case from Scotland, a patient presented with signs 
of pubertal development at 1 year of age [25]. This case was found to have the D578Y mutation (equivalent to D590Y in eelLHR), which is the mutant that reduces the highest basal level of cAMP production in transfected CHO-K1 cells. The residues Met-410, Leu-469, Asp-590, Asp383, and Tyr-546 in eelLHR are conserved among LHRs, suggesting that these are important for normal receptor function. Receptor mutations correlating to these residues in eelLHR have not previously been described.

In the present study, we show that the three activating mutations in eelLHR resulted in markedly increased cAMP stimulation under basal condition, suggesting that these mutation cause constitutive activation of eelLHR, as also seen in the FMPP-causing mutations in hLHR. Compared to eelLHR-WT, the eelLHR-M410T, -L469R, and -D590Y mutants produced a 4-, 19.1-, and 7.8-fold increase, respectively, in basal cAMP production in CHO-K1 cells, indicating that these mutants were constitutively active, as previously reported in mammalian LHRs [9,17,24,26,27]. Cells expressing the hLHR-M398T mutant exhibited high basal cAMP levels (Yano et al., 1996). The same mutation has been detected in an FMPP patient and in the patient's mother and brother [17]. This mutation is of special interest, since one member of this family has the mutation, with no evidence of precocious puberty [18]. The basal levels of cAMP production increased approximately 15- to 25-fold in the M398T mutant receptor compared to WT receptor [5]. In this paper, we also describe a constitutively activating mutation in eelLHR, M410T, which is located in the same second transmembrane region. The L457R mutation (equivalent to L469R in eelLHR) was the first activating mutation identified in hLHR and cells expressing this mutant receptor exhibit significantly higher basal cAMP accumulation (7-to 14-fold) than cells expressing the WT receptor [9]. We have also reported that cells expressing rLHR-L435R (equivalent to L469R in eelLHR) display a 47-fold increase in basal cAMP responses and do not react to hCG with a further stimulation in cAMP accumulation [24]. In the hLHR, the complex form between hLHR-L457R and hCG is not migrated to the lysosomes, but most of it is returned to the cell surface and hormone degradation is hardly detectable [26]. These results are consistent with our current data, showing that the L469R mutant of eelLHR remarkably increases (19.1fold) the basal cAMP response in the absence of agonist. But the maximal cAMP response was approximately 53\% lower than that of the WT receptor as previously described in hLHR [24]. 
Thus, our data suggested that the constitutively active mutant, L469R, was easily distinguishable from agonist-activated eelLHRs analyzed in this study.

The aspartic acid residue at position 590 is conserved in all LH receptors, including eelLHR, but is not found in any other GPCR. The D578Y mutant (equivalent to D590Y in eelLHR) was first reported to be inherited in an autosomal dominant and is associated with signs of puberty by 4 years of age. This mutant hLHR results in a 4.5-fold increase cAMP production under basal condition, with an $\mathrm{EC}_{50}$ similar to that of the WT receptor. Agonist-independent stimulation of cAMP production by this mutant receptor represented $42 \%$ of the maximal stimulation [6]. This is consistent with our results showing that the eelLHR-D590Y mutation, at a conserved in glycoprotein hormone receptor site, resulted in constitutive receptor activity.

Kosugi et al. [12] suggested that the $\mathrm{Asp}^{578}$ side chain in hLHR has the appropriate position to recognize as a hydrogen bond acceptor and is important for vitalizing the inactive state of LHR. Other constitutively activating mutations (Ile542Leu, Asp564Gly, Met571Ile, and Cys581Arg) have been identified by analyzing genomic DNA from 32 unrelated FMPP [13]. These sites are conserved among glycoprotein hormone receptors, suggesting an important role in receptor function. These data suggest that the region spanning nucleotides 1624-1741 of exon 11 is a hotspot for heterogeneous point mutations that activate hLHR and cause FMPP. The rat LHRD556Y mutant also exhibits a 25-fold increase in basal cAMP production, but responds to hCG with a normal increase in cAMP accumulation [24]. Based on the model of activation and the observations summarized above, we expected that eelLHR mutations that induce constitutive activation would result in specific changes to the receptor-ligand complex. The D590Y mutant induced an elevated basal cAMP level corresponding to approximately $10 \%$ of the maximal cAMP response. However the L469R mutant induced a highly elevated basal cAMP level corresponding to approximately $44 \%$ of the maximal cAMP response. These results suggested that the configurations of these three mutants are different in the signal transduction of maximal cAMP responses by $\mathrm{LH}$.

In the inactive mutants, highly conserved amino acids present in the second TM helix (codon 383) and in the fifth TM helix (codon 546) were mutated to asparagine and phenylalanine, respectively. As predicted from results obtained with other GPCRs [23,24,28,29], these mutations (eelLHR-D383N and eelLHR-Y546F) were expected to impair signal transduction. 
Cells expressing rLHR-D383N display a rightward shift in the $\mathrm{EC}_{50}$ for cAMP stimulation, but a normal maximal response [23]. The Y524F mutant in rLHR was also a signaling-impairing mutation. Cells expressing this mutant respond a normal level of cAMP in the absence of agonist, but their maximal response is only $14 \%$ lower than that of the WT receptor [24]. These results are consistent with our data, showing that $\mathrm{D} 383 \mathrm{~N}$ and $\mathrm{Y} 546 \mathrm{~F}$ are signaling-impairing mutations in eelLHR. The maximal response by these mutant receptors was only 24-32\% of the maximal response of eelLHR-WT. The internalization in inactivation mutants, rLHR-D383N and rLHRY524F, was much slower than in rLHR-WT [23]. In the present study, D383N and Y546F mutations were predicted to induce the inactivation of eelLHR. However, these mutations did not completely impair signal transduction in eelLHR. Thus, we suggest that these mutations are system-dependent or species-specific and therefore, may not have the same effects in fish systems.

\section{Experimental Section}

\subsection{Materials}

The pGEMTeasy cloning vector was purchased from Promega (Madison, WI, USA). The mammalian expression vector, pcDNA3, FreeStyle MAX transfection reagent, FreeStyle CHO-S suspension cells, and Lipofectamine-2000 were obtained from Invitrogen (Carlsbad, CA, USA). Ham's F-12 medium, CHO-S-SFMII medium, and fetal bovine serum, OptiMEM medium were bought from Gibco BRL (Grand Island, NY, USA). CHO-K1 cells were obtained from the Japanese Cancer Research Resources Bank (JCRB, Tokyo, Japan). A homogeneous timeresolved fluorescence (HTRF) cAMP assay kit was purchased from Cisbio (Codolet, France). Monoclonal antibodies (5A11, 11A8, and 14F5) and rec-eelLH from CHO-K1 cells were produced in our lab as previously reported [30]. Rec-eelLH produced using baculovirus expression system was kindly donated by Dr. Sun-Me Hong (Institute of Gyeongbuk Marine Bio-Industry). The horseradish peroxidase (HRP) labeling of 8A11 monoclonal antibody was generously performed by Medexx Inc. (Seongnam, Korea). EelLHR cDNA was cloned from eel ovaries and testes as previously reported [31]. Polymerase chain reaction (PCR) and endonucleases reagents were from Takara (Osaka, Japan). QIAprep-Spin plasmid kits were bought from Qiagen Inc. 
(Hilden, Germany). Oligonucleotides were synthesized by Genotech (Dajeon, Korea). Disposable spinner flasks were purchased from Corning Inc. (Corning, NY, USA). Fetal bovine serum was bought from Hyclone Laboratories (Logan, UT, USA). Centrifugal Filter Devices were from Amicon Bio (Billerica, MA, USA). All other reagents used were from Sigma-Aldrich (St. Louis, MO, USA) or Wako Pure Chemicals (Osaka, Japan).

\subsection{Site-directed mutagenesis of activation and inactivation sites}

An overlap extension PCR strategy was used to create activating and inactivating mutants in eelLHR cDNA, as previously described [32]. Two different sets of PCR were performed. In step 1 , forward and reverse primers (mutation primer) were used to amplify the first fragment. The second fragments were then amplified with forward (mutation primer) and reverse primers. In step 2, the amplified products (first and second fragments) from step 1 were used as templates to amplify the completely mutated fragments. The primer sequences used in these experiments are shown in Table 1. The full-length PCR product synthesized in step 2 was eluted and cloned into a pGEMTeasy vector. This clone was used to transform DH5 $\alpha$ competent cells. Plasmids were extracted and sequenced to confirm the presence of the mutations. A schematic representation of the mutations is shown in Fig. 1. We selected naturally occurring mutation sites for three activating (M410T, L469R, and D590Y) and two inactivating (D383N and Y546F) mutations in eelLHR.

\subsection{Vector construction}

cDNAs encoding WT and mutants eelLHR were digested with EcoRI and XhoI enzymes. The resulting fragments were then ligated into the eukaryotic expression vector, pcDNA3, as previously described $[31,33]$. Plasmids were then purified and the presence of the correct insert was confirmed by digestion with EcoRI and XhoI restriction enzymes. Finally, we constructed a total of six receptor genes, including eelLHR-WT (designated as pcDNA3-eelLHR-WT, pcDNA3-M410T, pcDNA3-L469R, pcDNA3-D590Y, pcDNA3-D383N, and pcDNA3-Y546F).

\subsection{Transient transfection}


CHO cells were transfected using the liposome transfection method, as previously described [32]. CHO cells were cultured in growth medium (Ham's F-12 medium containing 50 U/mL penicillin, $50 \mu \mathrm{g} / \mathrm{mL}$ streptomycin, $2 \mathrm{mM}$ glutamine, and $10 \%$ fetal bovine serum). CHO cells were grown to 80-90\% confluence in 6-well plates and plasmid DNAs were transfected using Lipofectamine reagent. After combining the diluted DNA with Lipofectamine samples were incubated for $20 \mathrm{~min}$. CHO cells were washed with Opti-MEM and the DNA-Lipofectamine complex was added to each well. After $5 \mathrm{~h}$, CHO growth medium containing $20 \%$ fetal bovine serum was added to each well. The cells were used for cAMP analysis at $48 \mathrm{~h}$ after transfection.

For ligand production, a rec-eelLH expression vector was transfected into CHO-suspension (CHO-S) cells using the FreeStyle MAX reagent transfection method, according to the manufacturer's instructions. One day prior to transfection, CHO-S cells were passaged at $5 \times 10^{5}$ cells/mL. Flasks were placed on an orbital shaking platform, rotating at $120-135 \mathrm{rpm}$ at $37^{\circ} \mathrm{C}$ in a humidified atmosphere of $8 \% \mathrm{CO}_{2}$ in air. On the day of transfection, the cell density was approximately $1.2-1.5 \times 10^{6}$ cells $/ \mathrm{mL}$. Next, $260 \mu \mathrm{g}$ of plasmid DNA was diluted int OptiPRO ${ }^{\mathrm{TM}}$ serum-free medium (SFM) to a total volume of $4 \mathrm{~mL}$. In a separate tube, $260 \mu \mathrm{L}$ of FreeStyle ${ }^{\mathrm{TM}}$ MAX Reagent was diluted in Opti-PRO ${ }^{\text {TM }}$ SFM to a total volume of $4 \mathrm{~mL}$ and mixed gently by inverting the tube. After the solutions were mixed, the DNA-FreeStyle ${ }^{\mathrm{TM}}$ MAX mix was incubated for $10 \mathrm{~min}$ at RT to allow complexes to form. DNA-FreeStyle ${ }^{\mathrm{TM}} \mathrm{MAX}$ Reagent complexes were slowly added to $200 \mathrm{~mL}$ of medium containing cells. Transfected cell cultures were incubated at $37^{\circ} \mathrm{C}$ in a humidified atmosphere of $8 \% \mathrm{CO}_{2}$ on an orbital shaking platform, rotating at $135 \mathrm{rpm}$. Finally, culture media were collected on day 7 after transfection and centrifuged at $100,000 \times \mathrm{g}$ for $10 \mathrm{~min}$ at $4^{\circ} \mathrm{C}$ to remove cell debris. Supernatants were collected and frozen at $-80^{\circ} \mathrm{C}$. The samples were concentrated using a Centricon filter or by freeze-drying and mixed with PBS. The concentration of rec-eelLH was analyzed by an enzyme-linked immunosorbent assay (ELISA), previously developed in our laboratory (Kim et al., 2016).

\subsection{ELISA analysis of rec-eelLH protein}

Rec-eelLH was quantified using a double-sandwich ELISA performed in plates coated with the monoclonal antibody, 5A11, which is directed against the $\alpha$-subunit of eelLH as described previously [30]. The standard of rec-eelLH was produced from E. coli and purified by 
performing Ni-NTA Sepharose column chromatography with $250 \mathrm{mM}$ imidazole. The wells were blocked by incubation with $1 \%$ skim milk in PBS for $1 \mathrm{~h}$ at $37^{\circ} \mathrm{C}$. After removal of the blocking reagent, the wells were washed with filtered PBS containing 0.05\% Tween 20 (PBS-T). Next, $100 \mu \mathrm{L}$ of rec-eelLH sample was added to the wells and incubated for $1-2 \mathrm{~h}$ at $37^{\circ} \mathrm{C}$. After washing three times with PBS-T, a 400-fold diluted, HRP-conjugated anti-eel 11A8 antibody in PBS was added and plates were incubated for $1 \mathrm{~h}$ at room temperature. The wells were washed 5 times and incubated with $100 \mu \mathrm{L}$ of substrate solution (tetramethylbenzidine) for 20 min at room temperature. The reaction was stopped by adding $50 \mu \mathrm{L}$ of $1 \mathrm{M} \mathrm{H}_{2} \mathrm{SO}_{4}$. Absorbance at $450 \mathrm{~nm}$ was measured in each well using a microplate reader (Cytation 3; Biotek, Winooski, VT, USA).

\section{6. cAMP analysis by homogeneous time-resolved fluorescence (HTRF)}

cAMP accumulation in CHO-K1 cells expressing eelLHR-WT and eelLHR mutants was measured using cAMP Dynamic 2 competitive immunoassay kits (Cisbio Bioassays, Codolet, France), as described previously [31]. In briefly cells transfected with eelLHR-WT and eelLHR mutants were added at 10,000 cells per well into a 384-well plate. Cells were stimulated by incubation with the agonist for $30 \mathrm{~min}$ at room temperature. The assay was terminated by incubation with the Cisbio detection reagents, cAMP-d2 and anti cAMP-cryptate (diluted 5-fold in lysis buffer and used at $5 \mu \mathrm{L} /$ well), for $1 \mathrm{~h}$ at room temperature. cAMP was detected by measuring the decrease in HTRF energy transfer $(665 \mathrm{~nm} / 620 \mathrm{~nm})$ using an Artemis K-101 HTRF microplate reader (Kyoritsu Radio, Tokyo, Japan). This method is a competitive immunoassay between native cAMP produced by cells and cAMP labeled with the dye, d2. Tracer binding is visualized using a monoclonal anti-cAMP antibody labeled with Eu3+Cryptate. The specific signal-Delta $\mathrm{F}$ (energy transfer) is inversely proportional to the concentration of cAMP in the standard or sample. Results were calculated from the $665 \mathrm{~nm} / 620 \mathrm{~nm}$ ratio and expressed as Delta F\% (cAMP inhibition), according to the following equation: [Delta $\mathrm{F} \%=$ (standard or sample ratio-sample negative) $\times 100 /$ ratio negative]. The cAMP concentrations for Delta F\% values were calculated using Prism software (GraphPad, Inc., La Jolla, CA, USA).

\subsection{Data analysis}


The Multalin interface-multiple sequence alignment tool was used for sequence analysis. GraphPad Prism 6.0 was used for the analysis of cAMP production and Grafit 5.0 (Erithacus Software Limited, Surrey, UK) was used for cAMP EC 50 value and stimulation curve analyses. Curves fitted in a single experiment were normalized to the background signal measured for mock-transfected cells. Each curve was drawn using data from at least three independent experiments.

\section{Conclusions}

In summary, our mutagenesis data suggest that the constitutive activation mutations (M410T, L469R, and D590Y) of eelLHR result in a significant increase in basal cAMP production, but respond to rec-eelLH with a concentration dependent increase in cAMP accumulation, as reported for mutations of these highly conserved amino acids in mammalian LHRs. However, the inactivation mutants (D383N and Y546F) were almost completely abolished in hormoneinduced receptor activation. However, these mutations did not totally impair in signal transduction of cAMP responsiveness. Thus, we suggest that the activation process involves an agonist-induced conformational change in the receptor. The mechanism whereby the constitutively active mutants result in a significant increase in the basal cAMP response and inactivation mutants impair signal transduction require further investigation. Future studies using these glycoprotein hormone receptors could provide important information regarding the structure-function relationship of GPCRs in signal transduction.

\section{Acknowledgements}

This work was supported by the Korean Research Foundation Program (2018R1A2B6007794), Republic of Korea.

\section{Author contributions}

M.B performed the experiment. D-A.K was financially support. D-J.K, S-M.H, M-H.K and K-S.M conceived the study, designed the experiment and drafted the manuscript. All authors read and approved the final manuscript. 


\section{Conflicts of Interest}

The authors have no financial or personal relationship that could inappropriately influence this research.

\section{References}

1. Kudo, M., Osuga, Y., Kobilka, B.K., Hsueh, A.J. Transmembrane region V and VI of the human luteinizing hormone receptor are required for constitutive activation by a mutation in the third intracellular loop. J. Biol. Chem. 1996, 271, 22470-22478.

2. Ascoli, M., Segaloff, D.L. On the structure of the luteinizing hormone/chorionic gonadotropin receptor. Endoc. Rev. 1989, 10, 27-44.

3. Segaloff, D.L., Ascoli, M. The lutropin/choriogonadotropin receptor... 4 years later. Endoc. Rev. 1993, 14, 324-347.

4. Meehan, T.P., Narayan, P. Constitutively active luteinizing hormone receptor: consequences of in vivo expression. Mol. Cell Endocrinol. 2007, 260-262, 294-300.

5. Kraaij, R., Post, M., Kremer, H., Milgrom, E., Epping, W., Brunner, H.G., Grootegoed, J.A., Themmen, A.P. A missense mutation in the second transmembrane segment of the luteinizing hormone receptor causes familial male-limited precocious puberty. J. Clin. Endocrinol. Metab. 1995, 80, 3168-3172.

6. Shenker, A., Laue, L., Kosugi, S., Merendino, J.J., Minegishi, T., Cutler, G.B.Jr. A constitutively activating mutation of the luteinizing hormone receptor in familial male precocious puberty. Nature 1993, 365, 652-654.

7. Yano, K., Kohn, L,D., Saji, M., Kataoka, N., Okuno, A., Cutler, G.B.Jr. A case of malelimited precocious puberty caused by a point mutation in the second transmembrane domain of the luteinizing hormone choriogonadotropin receptor gene. BBRC. 1996, 220, 1036-1042.

8. Wu, S.M., Leschek, E.W., Rennert, O.M., Chan, W.Y. Luteinizing hormone receptor mutations in disorders of sexual development and cancer. Front Biosci. 2000, 5, 343-352.

9. Latronico, A.C., Chai, Y., Arnhold, I.J., Liu, X., Mendonca, B.B., Segaloff, D.L. A homozygous microdeletion in helix 7 of the luteinizing hormone receptor associated with familial testicular and ovarian resistance is due to both decreased cell surface expression and impaired effector activation by the cell surface receptor. Mol. Endocrinol. 1998, 12, 442450.

10. Narayan, P. Genetic models for the study of luteinizing hormone receptor function. Front Endocrinol. 2015, 6, 152

11. Shenker, A. Activating mutations of the lutropin choriogonadotropin receptor in precocious puberty. Receptors Channels 2002, 8, 3-18.

12. Kosugi, S., Mori, T., Shenker, A. The role of $\mathrm{Asp}^{578}$ in maintaining the inactive conformation of the human lutropin/choriogonadotropin receptor. J. Biol. Chem. 1996, 271, 31813-31817.

13. Laue, L., Chan, W.Y., Hsueh, A.J.W., Kudo, M., Hsu, S.Y., Wu, S.M., Blomberg, L., Cutler, G.B.Jr. Genetic heterogeneity of constitutively activating mutations of the human luteinizing hormone receptor in familial male-limited precocious puberty. Proc. Natl. Acad. Sci. USA 
1995, 92, 1906-1910.

14. Kosugi, S., Mori, T., Shenker, A. An anionic residue at position 564 is important for maintaining the inactive conformation of the human lutropin/choriogonadotropin receptor. Mol. Pharmacol. 1998, 53, 894-901.

15. Laue, L., Wu, S.M., Kudo, M., Bourdony, C.J., Cutler, G.B.Jr., Hsueh, A.J., Chan, W.Y. Compound heterozygous mutations of the luteinizing hormone receptor gene in Leydig cell hypoplasia. Mol. Endocrinol. 1996, 10, 987-997.

16. Ignacak, M., Hilczer, M., Zarzycki, J., Trzeciak, W.H. Substitution of M398T in the second transmembrane helix of the LH receptor in a patient with familial male-limited precocious puberty. Endocrine J. 2000, 47, 595-599.

17. Ignacak, M., Niedziela, M., Trzeciak, W.H. Transition C2718T in the AR gene, resulting in generation of a termination codon and truncated form of the androgen receptor, causes complete androgen insensitivity syndrom. J. Appl. Genet. 2002, 43, 109-114.

18. Evans, B.A., Bowen, D.J., Smith, P.J., Clayton, P.E., Gregory, J.W. A new point mutation in the luteinizing hormone receptor gene in familial and sporadic male limited precocious puberty: genotype does not always correlate with phenotype. J. Med. Genet. 1996, 33, 143147.

19. Richter-Unruh, A., Wessels, H.T., Menken, U., Bergmann, M., Schmittmann-Ohters, K., Schaper, J., Tappeser, S., Hauffa, B.P. Male LH-independent sexual precocity in a 3.5-yearold boy caused by a somatic activating mutation of the LH receptor in a Leydig cell tumor. $J$. Clin. Endocrinol. Metab. 2002, 87, 1052-1056.

20. Boot, A.M., Lumbroso, S., Verhoef-Post, M., Richter-Unruh, A., Looijenga, L.H., Funaro, A., Beishuizen, A., van Marle, A., Drop, S.L., Themmen, A.P. Mutation analysis of the LH receptor gene in Leydig cell adenoma and hyperplasia and functional and biochemical studies of activating mutations of the LH receptor gene. J. Clin. Endocrinol. Metab. 2011, 96, 1197-1205.

21. Huhtaniemi, I.T., Themmen, A.P. Mutations in human goandotropin and gonadotropinreceptor gene. Endocrine 2005, 26, 207-217.

22. Themmen, A.P. An update of the pathophysiology of human goandotropin subunit and receptor gene mutations and polymorphisms. Reproduction 2005, 130, 263-274.

23. Dhanwada, K.R., Vijapurkar, U., Ascoli, M. Two mutations of the lutropin/ choriogonadotropin receptor that impair signal transduction also interfere with receptormediated endocytosis. Mol. Endocrinol. 1996, 10, 544-554.

24. Min, K.S., Liu, X., Fabritz, J., Jaquette, J., Abell, A.N., Ascoli, M. Mutations that induce constitutive activations and mutations that impair signal transduction modulate the basal and/or agonist-stimulated internalization of the lutropin/choriogonadotropin receptor. J. Biol. Chem. 1998, 273, 34911-34919.

25. Bavovic-Vuksanovic, D., Donaldson, M.D., Gibson, N.A., Wallace, A.M. Hazards of ketoconazole therapy in testotoxicosis. Acta Paediatr. 1994, 83, 994-997.

26. Galet, C., Ascoli, M. A constitutively active mutant of the human lutropin receptor (hLHRL457R) escapes lysosomal targeting and degradation. Mol. Endocrinol. 2006, 20, 29312945. 
27. Latronico, A.C., Segaloff, D.L. Insights learned from L457R, an activating mutant of the human lutropin receptor. Mol. Cell Endocrinol. 2007, 260-262, 287-293.

28. Ji, I., Ji, T.H. Asp383 in the second transmembrane domain of the lutropin receptors is important for high affinity hormone binding and cAMP production. J. Biol. Chem. 1991, 266, 4953-14957.

29. Quintana, J., Wang, H., Ascoli, M. The regulation of the binding affinity of the luteinizing hormone/choriogonadotropin receptor by sodium ions is mediated by a highly conserved aspartate located in the second transmembrane domain of $\mathrm{G}$ protein-coupled receptors. Mol. Endocrinol. 1993, 7, 767-775.

30. Kim, D.J., Park, C.W., Byambaragchaa, M., Kim, S.K., Lee, B.I., Hwang, H.K., Myeong, J.I., Hong, S.M., Kang, M.H., Min, K.S. Data on the characterization of follicle-stimulating hormone monoclonal antibodies and localization in Japanese eel pituitary. Data Brief 2016, 8, 404-410.

31. Byambaragchaa, M., Kim, D.J., Kang, M.H., Min, K.S. Site specificity of eel luteinizing hormone N-linked oligosaccharides in signal transduction. Gen. Comp. Endocrinol. 2018, 268, 50-56.

32. Min, K.S., Hiyama, T., Seong, H.H., Hattori, N., Tanaka, S., Shiota, K. Biological activities of tethered equine chorionic gonadotropin (eCG) and its deglycosylated mutants. J. Reprod. Dev. 2004, 50, 297-304.

33. Kim, D.J., Park, C.W., Kim, D.W., Park, H.K., Byambaragchaa, M., Lee, N.S., Hong, S.M., Seo, M.Y., Kang, M.H., Min, K.S. Production and characterization of monoclonal antibodies against recombinant tethered follicle-stimulating hormone form Japanese eel anguilla japonica. Gen. Comp. Endocrinol. 2016, 233, 8-15

\section{Figures Legends}

Figure 1. Total cAMP levels stimulated by rec-eelLH in CHO-K1 cells transfected with constitutively active eelLHR mutants. CHO-K1 cells transiently transfected with eelLHR-WT and mutant eelLHRs (M410T, L469R, and D590Y) were stimulated with rec-eelLH (0-1,000 $\mathrm{ng} / \mathrm{mL}$ ) for $30 \mathrm{~min}$. Finally, the cAMP production was detected using homogenous time-resolved fluorescence (HTRF) assay. The cAMP accumulation stimulated by rec-eelLH is shown as Delta F\%. cAMP concentration was calculated using GraphPad Prism software. 
Figure 2. Rec-eelLH-stimulated cAMP production in CHO-K1 cells transfected with inactivating eelLHR mutants. CHO-K1 cells transiently transfected with eelLHR-WT and mutant eelLHRs (D383N and Y546F) were stimulated with rec-eelLH (0-1,000 ng/mL) for 30min. Total cAMP accumulation was analyzed using a homogenous time-resolved fluorescence (HTRF) assay. The empty circles denote wild-type eelLHR and black circles denote the mutants. Each point is the mean of duplicate experiments and one result from three similar experiments is presented.

Figure 3. Schematic representation of the eelLHR structure. The location of the three constitutively activating mutations (M410T, L469R, and D590Y) and the two inactivating mutations (D383N and Y546F) are indicated in the structure of eelLHR. Amino acid sequences at the mutated sites in the transmembrane domains of eelLHR are shown. EelLHR sequence alignment was performed with homologous mammalian LH/CGR sequences obtained from the NCBI database. The activating and inactivating sites were determined by comparison with the corresponding sites in eelLHR. Red circles indicate constitutively activating mutations and the blue circle indicate inactivating mutations. EC, extracellular domain; TM, transmembrane domain; IC, intracellular domain.

Table 1. List of primers used to construct eelLHR mutants

\begin{tabular}{|c|c|c|}
\hline & Primer name & Primer sequence \\
\hline 1 & eelLHR-wt forward & $\begin{array}{l}\text { 5'-ATGAATTCATGTCCAATCTGCTCTTGTGGACGATG-3' } \\
\text { EcoRI site }\end{array}$ \\
\hline 2 & eelLHR-wt reverse & $\begin{array}{l}\text { 5'- CCTCGAGTTATTTAGGACCTCTGTTGAGAAT-3' } \\
\text { XhoI site }\end{array}$ \\
\hline 3 & M41 OT forward & 5'- TCTCCCGCTTCCTCACGTGCAACCTGGCCTT-3' \\
\hline 4 & M410T reverse & 5'-AAGGCCAGGTTGCACGTGAGGAAGCGGGAGA-3, \\
\hline 5 & L469R forward & 5'- TGTCCGTCTACACCCGGACCGTCATCACCCT -3, \\
\hline 6 & L469R reverse & 5'-AGGGTGATGACGGTCCGGGTGTAGACGGACA-3' \\
\hline 7 & D590Y forward & 5'- TGCTCATATTCACCTACTTCCTGTGCATGGC-3' \\
\hline 8 & D590Y reverse & 5'-GCCATGCACAGGAAGTAGGTGAATATGAGCA -3' \\
\hline 9 & $\mathrm{D} 383 \mathrm{~N}$ forward & 5'- CTGGCCTTCGCCAACCTCTGCATGGGC-3' \\
\hline 10 & D383N reverse & 5'- GCCCATGCAGAGGTTGGCGAAGGCCAG-3' \\
\hline 11 & Y546F forward & 5'- GTCTGCGTCTGCTTCGGCCGCATCTAC-3' \\
\hline 12 & Y546F reverse & 5'- GTAGATGCGGCCGAAGCAGACGCAGAC-3' \\
\hline
\end{tabular}

*Underlined nucleotides are the site of mutagenesis. 
Table 2. Bioactivity of eelLH receptors in cells expressing activating and inactivating receptor mutants

\begin{tabular}{|c|c|c|c|}
\hline \multirow[b]{2}{*}{ eelLH receptors } & \multicolumn{3}{|c|}{ cAMP responses } \\
\hline & $\begin{array}{c}\text { Basal } \\
\left(\mathrm{nM} / 10^{4} \text { cells }\right)\end{array}$ & $\begin{array}{l}\mathrm{EC}_{50} \\
\text { (ng) }\end{array}$ & $\begin{array}{c}\mathrm{Rmax} \\
\left(\mathrm{nM} / 10^{4} \text { cells }\right)\end{array}$ \\
\hline eelLHR-WT & $\begin{array}{l}1.2 \pm 0.5 \\
(1 \text {-fold })\end{array}$ & $\begin{array}{c}24.0 \pm 4.0 \\
(100 \%)\end{array}$ & $\begin{array}{c}96.0 \pm 6.2 \\
(100 \%)\end{array}$ \\
\hline eelLHR-D590Y & $\begin{array}{l}9.3 \pm 0.8 \\
(7.8 \text {-fold })\end{array}$ & $\begin{array}{c}152.1 \pm 5.2 \\
(15 \%)\end{array}$ & $\begin{array}{c}89.1 \pm 4.5 \\
(92 \%)\end{array}$ \\
\hline eelLHR-L469R & $\begin{array}{l}22.9 \pm 1.5 \\
(19.1 \text {-fold })\end{array}$ & $\begin{array}{r}2.9 \pm 0.1 \\
(827 \%)\end{array}$ & $\begin{array}{c}51.2 \pm 5.0 \\
(53 \%)\end{array}$ \\
\hline \multirow[t]{2}{*}{ eelLHR-M410T } & $\begin{array}{l}4.8 \pm 0.3 \\
(4.0 \text {-fold })\end{array}$ & $\begin{array}{c}118.0 \pm 5.2 \\
(20 \%)\end{array}$ & $\begin{array}{c}70.1 \pm 4.3 \\
(73 \%) \\
\end{array}$ \\
\hline & \multicolumn{3}{|c|}{ cAMP responses } \\
\hline eelLH receptors & $\begin{array}{c}\text { Basal } \\
\left(\mathrm{nM} / 10^{4} \text { cells }\right)\end{array}$ & $\begin{array}{c}\mathrm{EC}_{50} \\
(\mathrm{ng} / \mathrm{mL})\end{array}$ & $\begin{array}{c}\mathrm{Rmax} \\
\left(\mathrm{nM} / 10^{4} \text { cells }\right)\end{array}$ \\
\hline eelLHR-WT & $1.6 \pm 0.0$ & $\begin{array}{c}28.5 \pm 4.0 \\
(100 \%)\end{array}$ & $\begin{array}{c}72.1 \pm 5.6 \\
(100 \%\end{array}$ \\
\hline eelLHR-D383N & $1.2 \pm 0.1$ & $\begin{array}{c}13.5 \pm 5.3 \\
(211 \%)\end{array}$ & $\begin{array}{c}23.2 \pm 2.1 \\
(32 \%)\end{array}$ \\
\hline eelLHR-Y546F & $0.7 \pm 0.1$ & $\begin{array}{c}42.4 \pm 3.5 \\
(67 \%)\end{array}$ & $\begin{array}{c}17.7 \pm 0.7 \\
(24 \%)\end{array}$ \\
\hline
\end{tabular}

Values are the means $\pm \mathrm{SEM}$ of triplicate experiments. $\mathrm{EC}_{50}$ values were determine determined from the concentration-response curves from in vitro bioassays. 
Fig. 1.
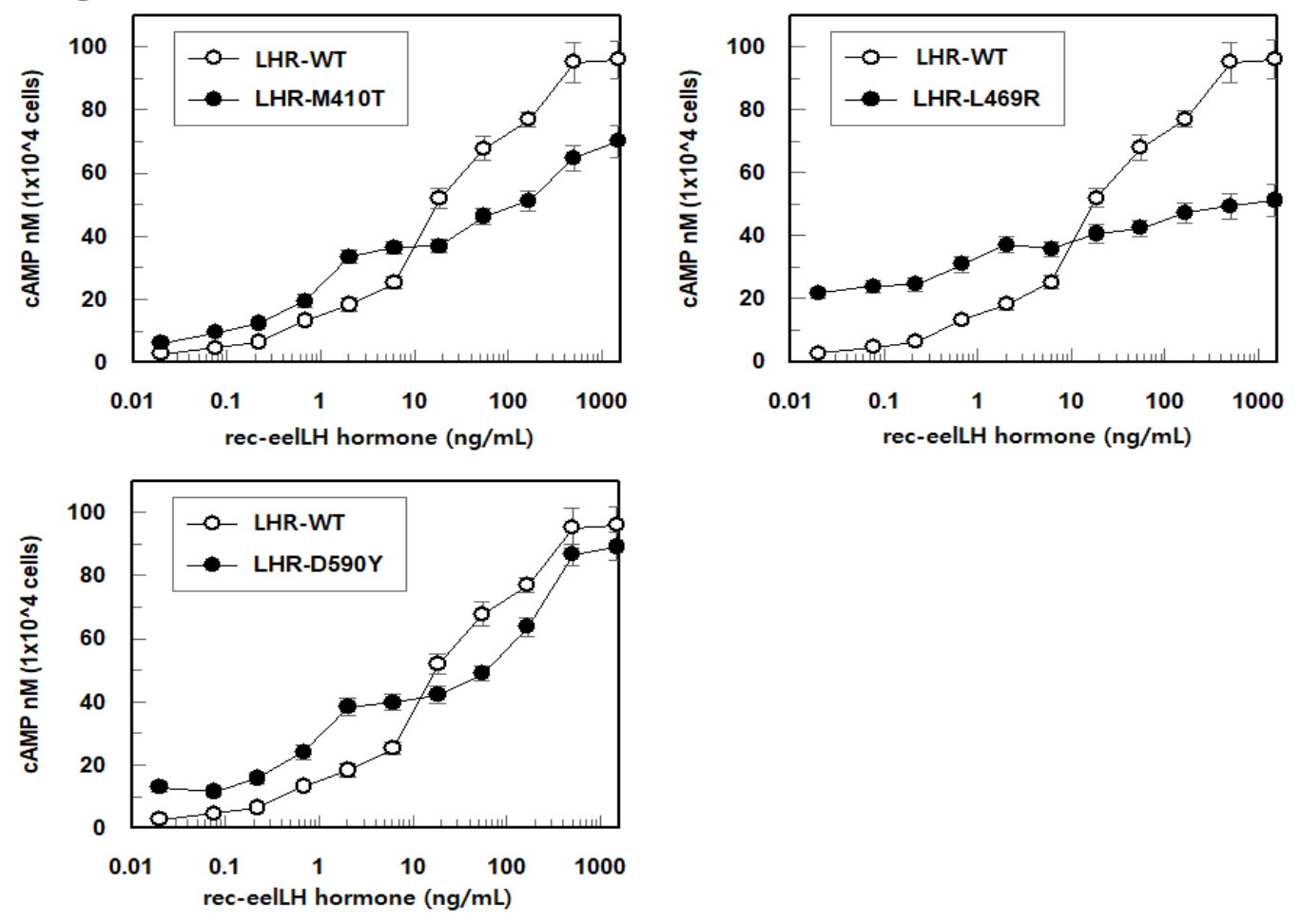

Fig. 2.
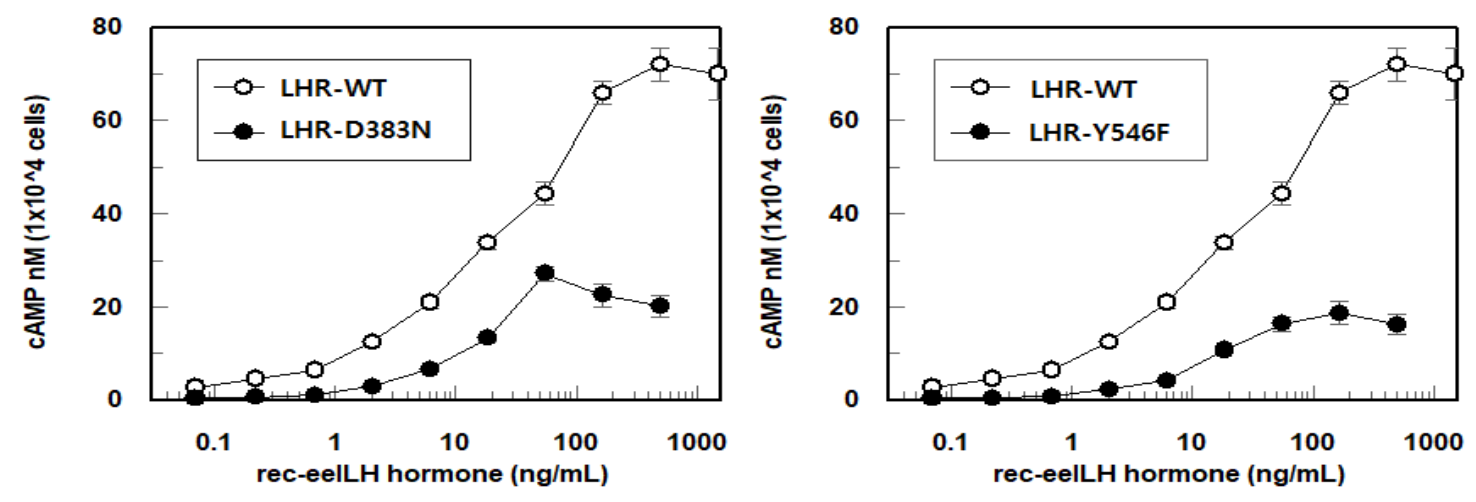
Fig. 3.

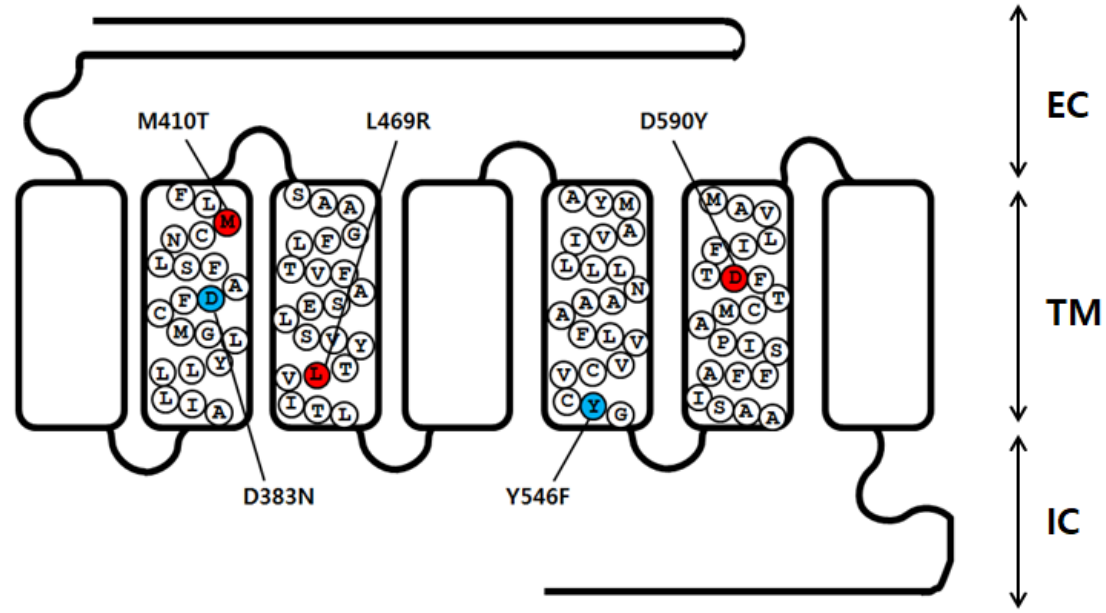

